\title{
Understanding Product State Relations within Manufacturing Processes
}

\author{
Benjamin Knoke, Thorsten Wuest, and Klaus-Dieter Thoben \\ Bremer Institut für Produktion und Logistik GmbH (BIBA), \\ Hochschulring 20, 28359 Bremen, Germany \\ $\{k n o$, wue, tho\}@biba.uni-bremen. de
}

\begin{abstract}
Today's manufacturing industry is forced to constantly improve its processes in order to stay competitive in a global market. Already highly optimized in many cases, academics and practitioners must identify furthur optimization potential by taking a closer look at the manufacturing process line. Within this paper, the product state based view is briefly introduced as a theoretical basis for the following analysis of relations between product state characteristics along the manufacturing process chain. First, the difference of dependencies and interdependencies are established through a time and process perspective. Possible dependencies are then classified, including theoretical examples. Concluding the paper, the requirements for a mapping of state characteristics are shown and possible opportunities of the presented approach are discussed.
\end{abstract}

Keywords: product state, manufacturing process, (inter-)dependencies, relations.

\section{Introduction}

When it comes to company requirements, manufacturing companies are experiencing more and more challenges from their customers towards product and information quality [1]. At the same time, the manufacturing processes themselves are becoming more complex, as they are no longer being carried out at one single location [2].

Business success of manufacturing companies is directly based on the quality of their processes, so there is a need for constant process improvement [3]. One step towards this goal is to increase the transparency of the processes, which in turn increases understanding of them. The product state based view focuses on describing an individual product along an industrial manufacturing process, including the state changes and information involved. It is based around the description of the product through its product state.

The product state within a manufacturing process can be described at any time with holistic knowledge about its relevant characteristics [4]. Despite this deterministic approach, holistic knowledge concerning all state characteristics is neither worthwhile nor feasible. The reasons for not considering a state characteristic can be divided into 
three groups [5]. They can either be technical (e.g. not measureable or measureable by destroying the product), financial (e.g. measurement is too costly), or caused by a knowledge gap (e.g. state characteristic is not known). However, some state characteristics can be characterized as relevant regarding their impact on the manufacturing process and the product state.

One way to identify relevant state characteristics is whether they include crucial information needed for each manufacturing process step [5]. Therefore, a product state characteristic that neither impacts the manufacturing process nor influences other product state characteristics may be disregarded. Knowing what the relevant state characteristics do can improves transparency and increases understanding of the manufacturing process itself. The state characteristics are often not independent, but relate to each other and form a complex (manufacturing) system, as well. This paper focuses on the understanding and structure of these relationships between product state characteristics.

\section{State Characteristics within Manufacturing Processes}

First explaining the problem with an initial modeling approach, this chapter then focuses on the relationships between state characteristics in manufacturing processes and in opportunities of application.

\subsection{Initial Modeling Approach}

A generalized model of a manufacturing process is shown in Fig. 1. Product states $(\mathrm{S})$ frame the process steps $(\mathrm{P})$, through which the product is then described by discrete product state characteristics (SC). The term relation describes the general connections between SCs. These relations can either be one-directional (dependent, [6]) or bidirectional (interdependent, [7]).

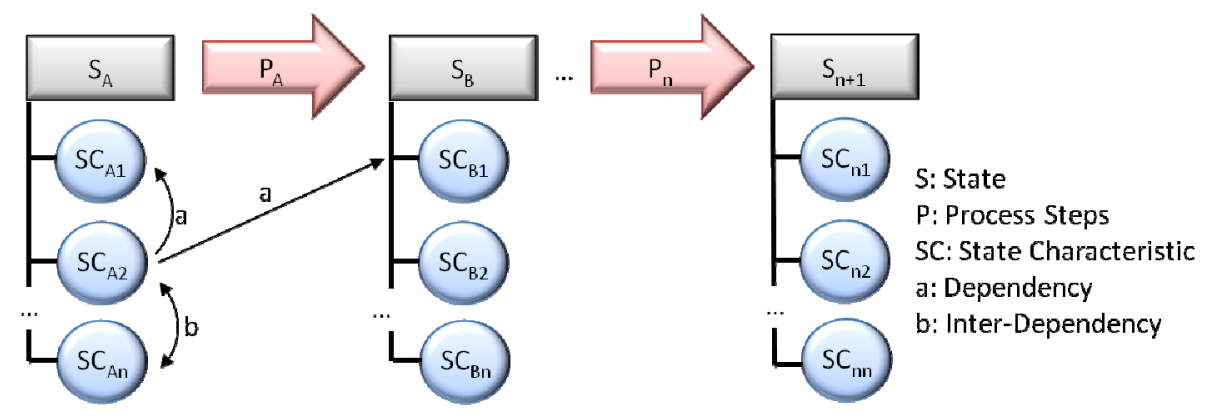

Fig. 1. Process model to visualize the relations between state characteristics 
The parameters of the manufacturing process also shape SCs. SCs depend partially on process parameters (e.g. cutting speed, damping pressure) of previous process steps. As shown in Fig. 1, these production steps are framed by previous and following states. The extent of the process steps has to be defined according to the modeling degree. It is possible to insert the product states into the process flow after each change of the product (or added value), but it is simpler and more reasonable to merge similar activities according to the modeling focus

The concept becomes significantly more complex through the integration of process parameters into it and will need to be elaborated in further research. An analytical approach towards the modeling of the relations between state characteristics is described in the following section.

\subsection{Characterization of Possible Relations between State Characteristics}

Interdependencies can only occur within a definite product state while dependencies cannot go against the process flow, so any potential shapes of the dependencies and interdependencies can be reduced. This is based on two axioms regarding the temporal restrictions of these connections:

- Dependencies can never go against the process flow, since a state characteristic always has an existing value that only past or present effects can influence.

- Interdependencies can only exist between state characteristics of the same state and time, since a future effect cannot impact the past.

If a decision within the manufacturing process is considered because of an upcoming event, it is in fact not influenced by the future event but by expected requirements and other information existing at the present time of the decision. For example: A car within a manufacturing process is painted red not because a customer is expected to react positively to this specific color at the moment of exchange, but because he had ordered a red car in the past, and this information was already useable during the manufacturing process.

\subsection{Analytical Approach and Different Types of State Characteristics}

As described within the previous section, a SC is also dependent on SCs from previous states. These cross-state relations can add up and become very complex. From an analytical perspective, the relations of SCs can be characterized as mathematical functions. For example, the dependency of a state characteristic SC1 on another state characteristic SC2 is expressed in the term SC1 = $\mathrm{f}$ (SC2). If interdependency between these two state characteristics exists, they are described by a common function $\mathrm{f}(\mathrm{SC} 1, \mathrm{SC} 2)$. These functions can be described either by a mathematical term (e.g. the mass of a cylinder: $\mathrm{m}=\rho * 1 * \mathrm{~d}^{2} * \pi$ ) or a text (e.g. the overall error ratio is $3 \%$ in the dayshift and $5 \%$ in the nightshift). 


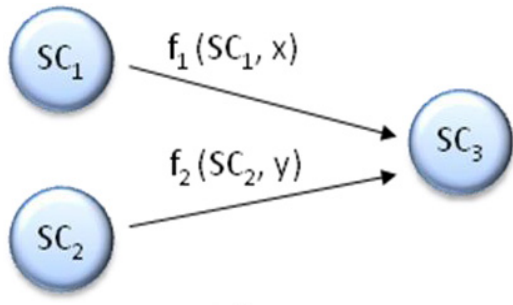

Discrete

Dependencies

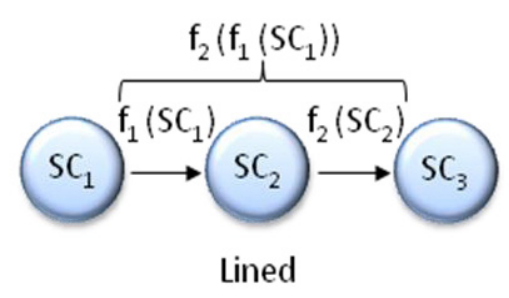

Dependencies

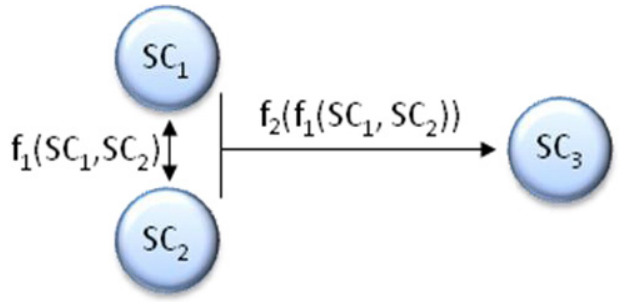

Linked

Dependencies

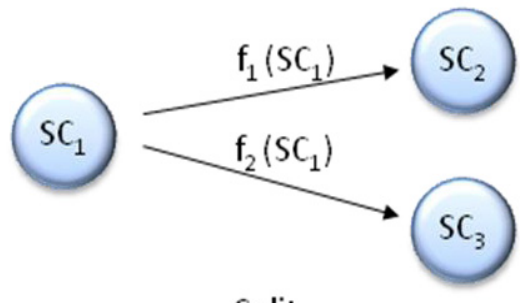

Split

Fig. 2. Possible characteristics of state characteristic dependencies

If dependencies between three or more state characteristics exist, four different characteristics can be identified. These types are visualized in Fig. 2. In complex models, these types may appear in combination:

- State characteristics with discrete dependencies have independent influence on another state characteristic. This occurs on the condition of additional process parameters $(\mathrm{x}, \mathrm{y})$. Since $\mathrm{SC}_{3}$ within the functions $\mathrm{SC}_{3}=\mathrm{f}_{1}\left(\mathrm{SC}_{1}\right)$ and $\mathrm{SC}_{3}=\mathrm{f}_{2}\left(\mathrm{SC}_{2}\right)$ could be eliminated, therefore $\mathrm{f}_{1}\left(\mathrm{SC}_{1}\right)=\mathrm{f}_{2}\left(\mathrm{SC}_{2}\right)$ would imply a direct connection. This causes the need of additional process parameters, which influence each function $\mathrm{SC}_{3}=\mathrm{f}_{1}\left(\mathrm{SC}_{1}, \mathrm{x}\right)$ and $\mathrm{SC}_{3}=\mathrm{f}_{2}\left(\mathrm{SC}_{2}, \mathrm{y}\right)$.

- Linked dependencies are another form of the connection between state characteristics. In this case, the combination of two or more state characteristics impacts another. If two state characteristics $\mathrm{SC}_{1}$ and $\mathrm{SC}_{2}$ influence $\mathrm{SC}_{3}$ within a linked dependency, they share an interdependency $\mathrm{f}_{1}\left(\mathrm{SC}_{1}, \mathrm{SC}_{2}\right)$, and $\mathrm{SC}_{3}$ can be described by the common function $\mathrm{SC}_{3}=\mathrm{f}_{1}\left(\mathrm{f}_{2}\left(\mathrm{SC}_{1}, \mathrm{SC}_{2}\right)\right)$.

- The sequence of multiple dependencies is defined as lined dependencies. If the dependencies $\mathrm{SC}_{2}=\mathrm{f}_{1}\left(\mathrm{SC}_{1}\right)$ and $\mathrm{SC}_{3}=\mathrm{f}_{2}\left(\mathrm{SC}_{2}\right)$ exist, they can be merged into a function $\mathrm{SC}_{3}=\mathrm{f}_{2}\left(\mathrm{f}_{1}\left(\mathrm{SC}_{1}\right)\right)$.

- Finally a state characteristic can also influence two or more other state characteristics. These split dependencies share a common origin and impact different state characteristics. E.g. the functions $\mathrm{SC}_{2}=\mathrm{f}_{1}\left(\mathrm{SC}_{1}\right)$ and $\mathrm{SC}_{3}=\mathrm{f}_{2}\left(\mathrm{SC}_{1}\right)$. 


$$
\mathrm{f}\left(\mathrm{SC}_{1}, \mathrm{SC}_{2}, \mathrm{SC}_{3}, \mathrm{SC}_{4}, \mathrm{SC}_{5}\right)
$$
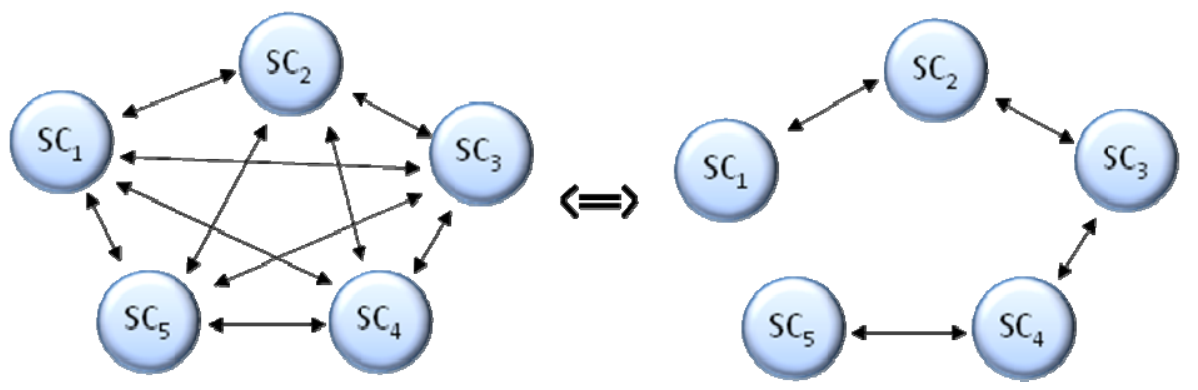

Fig. 3. Optional visualization possibilities of multiple interdependencies

If three or more state characteristics share interdependencies, they can be described by a common function. Following this approach, the visualization of all connections is redundant and can be replaced by a chain of interdependencies, as shown in Fig. 3. This can significantly improve the simplicity of a model. Mapping redundant information can be evaded by a structured approach to gather all relevant information. This crucial information is described in the next chapter.

\subsection{Crucial Information to Map State Characteristic Relations}

To benefit from the information of the relations between state characteristics and to share knowledge within a manufacturing process, a map of these relations needs to be modeled. To complete this task, certain information needs to be collected. The necessary data is:

- Aim and scope: Needs to be defined in order to create a model that considers relevant elements and neglects irrelevant ones.

- Modeling degree: Defines the modeled levels of relations. This includes the number of iterations in describing the relations of elements before and after being connected to state characteristics or process parameters.

- States and process steps: Along with their sequence, the states and process steps provide the basic structure of the process. Their sequential arrangement follows the rule of a bipartite graph, for a state is always followed by a process step and vice versa.

- Process parameters and state characteristics: The state characteristics and process parameters represent the nodes of the network. Each has to be aligned with the states and process steps.

- Transfer functions: The transfer functions describe the relations between state characteristic and process parameters. Collecting the transfer functions, that flow into each node to cover all relations, is sufficient.

A possible modeling form for a structured collection of all relevant data is shown in Table 1. Along with information about the manufacturing process and this data set, the relations of all state characteristics and process parameters are described. A model created on the basis of this data can be applied in multiple applications, which are described within the next section. 
Table 1. Exemplary structured modeling form to collect all relevant data

\begin{tabular}{|l|c|c|}
\hline \multicolumn{3}{|c|}{ Form of (process-name) } \\
\hline Aim & \multicolumn{2}{|c|}{ (aim of the model) } \\
\hline Scope & \multicolumn{2}{|c|}{ (scope and regarded relation levels) } \\
\hline $\begin{array}{l}\text { State or Process } \\
\text { Step }\end{array}$ & $\begin{array}{c}\text { State Characteristics or } \\
\text { Process Parameters }\end{array}$ & $\begin{array}{c}\text { Incoming functions of } \\
\text { relations }\end{array}$ \\
\hline \multirow{2}{*}{ A: (state A) } & (state characteristic A.1) & (function A.1.1) \\
\cline { 2 - 3 } & (state characteristic A.2) & (function A.2.1) \\
\hline \multirow{2}{*}{ 1: (process step 1) } & (process parameter 1.1) & (function 1.1.1) \\
\hline \multirow{2}{*}{ B: (state B) } & (state characteristic B.1) & (function B.1.1) \\
\cline { 2 - 3 } 2: (process step 2) & (process parameter 2.1) & (function B.1.2) \\
\hline \multirow{2}{*}{ C: (state C) } & (stanction 2.1.1) \\
\cline { 2 - 3 } & (state characteristic C.1) & (function C.1.1) \\
\hline 3: (process step 3) & (process parameter 3.1) & (function C.2.1) \\
\hline \multirow{2}{*}{ D: (state D) } & (state characteristic D.1) & (function D.1) \\
\cline { 2 - 3 } & (state characteristic D.2) & (function D.2.1) \\
\hline
\end{tabular}

\subsection{Opportunities for Application of the Concept}

The structure of the linked state characteristic concepts provides two different approaches for application. Whenever changes within a manufacturing process occur or have to be implemented, the model of state characteristic relations, when transferred to a PPC, can be applied. If the value of a state characteristic exceeds the acceptable range, the system can be used to create a model with all relevant influences on the state characteristic to identify the problem (Fig. 4, left). Alternatively, if a process parameter has to be changed, the system can be used for the opposite purpose: With a map that provides information about the impact of the change (Fig. 4, right).

A map that contains all information about any relation between state characteristics and process parameters within a manufacturing process tends to become very complex and difficult to handle. To solve this, a model with different hierarchical layers and levels of detail could be applied. One possible approach is to split the model into a meta-model and two sub-models:

- A meta-model that provides a general overview on all states and process steps with their aligned process parameters and state characteristics, along with the general process structure.

- A state-model that focuses on the relations of a single state or process step, and shows the relations of all process parameters or state characteristics of the focal state or process step. 


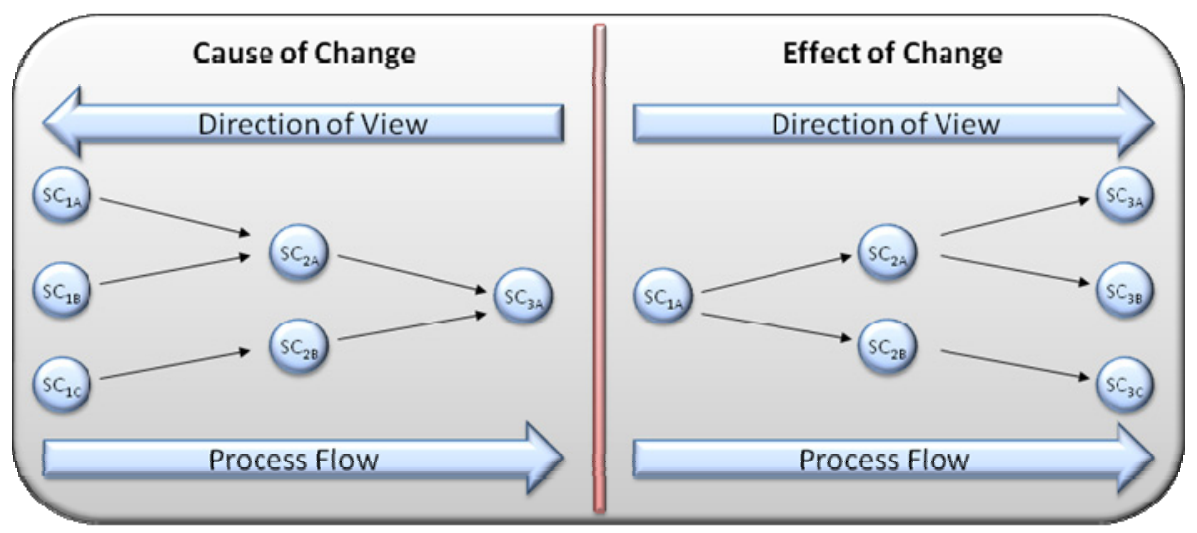

Fig. 4. Different opportunities of application

- A state characteristic-model that visualizes all relations of a single state characteristic or process parameter, and may include the functions that describe its relations.

With the information described within the previous section, and a defined modeling notation, the automatic generation of such models enters the realm of possibility and may be the outcome of future research.

\section{$2 \quad$ Limitation and Outlook}

This paper presented an approach to analyze and map relations between state characteristics based on the product state based view on collaborative manufacturing processes. After a brief introduction on the importance of transparency and in depth understanding of the own processes, the possible relations, dependencies and interdependencies were presented. The different types of dependencies between state characteristics were then elaborated on, and after a brief presentation on information requirements to apply the approach, the possible opportunities of the concept were discussed.

Overall, the topic of describing relations between state characteristics over a collaborative manufacturing process chain is very complex and, if applied in industry, requires an in-depth understanding and a high transparency of product, process and effects to realize its potential. Theoretically, if an application of the approach is possible, it will help to increase the final product quality and process efficiency by reducing waste and rework through early identification of problems and allocation of information to the right addressee.

The topic itself still needs further research concerning possible ways to identify and describe occurring relations in a practical and efficient way. Early practical insights into the manufacturing processes of an SME ( $1^{\text {st }}$ tier automotive supplier $)$ have indicated that the complexity of illustrating relations of state characteristics, along a manufacturing process, increases very fast and has to be managed very careful in order to not thwart the goal of increasing transparency for the stakeholders at hand. 
Due to these first impressions, parallel to further investigate the possibilities to describe relations on a cause-effect basis, other promising methods and tools such as combined cluster analysis and machine learning will be elaborated on, based on their contribution towards the goals of the product state based view on manufacturing processes.

\section{References}

1. Kovacic, M., Sarler, B.: Application of the Genetic Programming for Increasing the Soft Annealing Productivity in Steel industry. Materials and Manufacturing Processes 24, 369-374 (2009)

2. Seifert, M.: Unterstützung der Konsortialbildung in Virtuellen Organisationen durch perspektives Performance Measurement. Dissertation Universität Bremen. Bremen: Mainz Verlag (2007)

3. Linß, G.: Qualitätsmanagement für Ingenieure. München/Wien: Hanser Verlag (2002)

4. Wuest, T., Seifert, M., Klein, D., Thoben, K.-D.: Der Werkstückzustand im Informationsmanagement. Productivity Management 16 (2011)

5. Wuest, T., Klein, D., Thoben, K.-D.: State of steel products in industrial production processes. Procedia Engineering 10, S. 2220-S. 2225 (2011)

6. Farlex, Inc. (2009:2): TheFreeDictionary.com Großwörterbuch Deutsch als Fremdsprache (2009), Online-resource: http://de.thefreedictionary.com/abhängigkeit (last access on August 05, 2012)

7. Miller, G.A., et al.: Dictionary entry overview: What does interdependency mean? (2011), Online-resource: http: / /www . audioenglish.net/dictionary/ interdependency.htm (last access on August 05, 2012) 